\title{
Adverse drug reactions: monitoring, reporting and prevention
}

\author{
Sri Lanka is way behind. Prescribers, pharmacists and the CDDA should initiate and sustain the \\ remedial actions
}

\section{Introduction}

Pharmacovigilance is the process of being alert to the possible unwanted or harmful effects of therapeutic medications so that they could be detected early and remedial measures instituted (1). Adverse drug reaction (ADR) monitoring is one of the more effective forms of pharmacovigilance. An ADR is defined as 'an appreciably harmful or unpleasant reaction, resulting from an intervention related to the use of a medicinal product, which predicts hazard from future administration and warrants prevention or specific treatment, or alteration of the dosage regimen, or withdrawal of the product' (2). ADRs can be broadly grouped into three classes: dose related, allergic, and idiosyncratic. Dose related ADRs are predictable events that will take place in most if not all patients, provided a sufficiently high dose of medication is given. Allergic reactions have the following features: they occur in a small number of patients, require previous exposure to the same or a chemically related drug, develop rapidly after re-exposure, and produce clinical syndromes commonly associated with immunologic reactions (3). Idiosyncratic ADRs are unpredictable, will occur in only a few individuals and bear no relationship to the dose. ADR related admissions are increasing (4) and becoming a major concern worldwide, reaching levels of $68.7 / 1000$ admissions (5).

\section{Why monitor ADRs?}

New chemical entities (NCI), as well as new formulations of existing medications, are increasing at a rapid rate. NCIs are released to the market after phase 3 trials. In phase I trials the drug is tested on normal volunteers to determine their pharmacodynamic effects and possible toxic effects. In phase 2 the new compound is compared either with a placebo, or with an existing compound with similar pharmacological effects. Phase 3 trials involve a much larger number of patients, are carried out in several centres often situated in several countries. Even after phase 3 there is only limited experience with use of a new drug. Trials up to this point may be sufficient to detect ADRs that may occur with a relatively high frequency, but rare events may go undetected. Some of these will manifest as ADRs during phase 4 or post-marketing surveillance (6).

With better health care and socio-economic development life expectancy has increased, and the number of people in the older age groups has also increased (7). An ageing population will be more likely to have cardiovascular, puimonary, musculoskeletal and metabolic disorders that necessitate several medications. Recent studies have shown that ADRs are much more common in patients taking more than one medication (8). With advancing age physiological reserve is also reduced. This makes the older patients more vulnerable to the effect of ADRs compared to younger patients, and a greater degree of disability (9).
The economic impact of ADR-related admissions to the health care delivery system are substantial. Several studies have shown that $8 \%$ to $18 \%$ of all admissions are related to adverse effects. Some of these are to high dependency units. The average expenditure for such admissions are in the region of US $\$ 2000$ (10) and Euro 4150 (11) in developed countries. The limited resources of health care delivery systems in developing countries are stretched even further by ADR-related admissions.

\section{Monitoring systems for ADRs}

ADR monitoring systems can be classified as local, global and industry related systems. They supplement one another. A local system could be limited to one hospital or even to a few wards in larger hospitals. The information collected will be used at local level and also passed on to national and global monitoring centres

The pharmaceutical industry has its own well established ADR monitoring systems. At the time of release of a new product the innovator company must prepare a core data sheet pertaining to that product, taking into consideration the available data at the time of initial market authorisation. The core data sheet contains, among other data, the core safety information. This core safety information consists of safety information available up to the end of phase 3 trials and this is known as the initial core safety information. The Council for International Organizations of Medical Sciences working group III has set up proposals for producing and modifying the core data sheets and the core safety information it contains. The working group has recommended as its model the Summary of Product Characteristics (SPC), the official document of the European Union (12). As there is a high legal liability on the initial developers of a product the ADR monitoring systems of the pharmaceutical industry are efficient and well coordinated. It is mandatory in many countries for pharmaceutical companies or their authorised agents to submit ADR reports to the pharmacovigilance arm of the national regulatory authority. In most instances regulatory authorities have to be informed within 15 days of a serious adverse reaction detected by the company. ADR data collected by the national regulatory authority is analysed by them for possible signal detection. Likely signals are further evaluated by an expert committee to assess its true impact, and a report is formulated, which is submitted to the committee responsible for the evaluating drug registrations. This committee can use the information to modify the initial core safety information as appropriate to the local setting and prepare national data sheets.

The global monitoring centres will assimilate all information coming its way by way of individual reports, reports collected from collaborating centres, industry related monitoring and reports filed by licensing authorities. 
The global monitoring centres have the ability to detect signals early and to transmit these along with their recommendations to the licensing authorities world-wide. This information helps them decide on the issue of licenses to individual products $(13,14)$. The global monitoring centres also alert the industry about serious adverse events, so that the core safety information can be amended as soon as new ADRs are detected.

\section{Reporting of ADRs}

ADR reporting plays a key role in detection and prevention of ADRs, for it is only with adequate reporting that signals can be detected and preventive measures taken. The clinical staff have the largest reponsibility towards ADR reporting, as they are most likely to detect ADRs. They should consider ADR reporting as a part of their normal services and not as a special function. For reporting ADRs a designated form should be made available to the ward staff. It should be completed by the person filing the report and posted or delivered to the monitoring centre.

Unfortunately, there is a certain degree of reluctance on part of clinical staff to report ADRs (15) due to many reasons such as not knowing that they should be reported, and a lack of knowledge about the value of ADR reporting, time, necessary forms, and reinforcement from the ADR monitoring centres in the form of feedback on the reports sent by them. Larger institutions can overcome this problem to some extent by having a well identified ADR unit and an ADR team (16). The ADR team should consist of an identified officer in each reporting unit eg. registrar, the chief pharmacist (17) and ward pharmacist, and one or more clinical pharmacologists (18). They should regularly alert the clinical staff to the value of ADR reporting and promote it.

The pharmacist plays an important role in reporting and preventing ADRs. He should visit the wards and go through the patients' records and be vigilant of possible ADRs. He should not hesitate to discuss with ward staff when an ADR is likely to occur in particular clinical settings $(19,20)$. The clinical staff must be responsive and discuss with the pharmacist a possible alternative therapy if they feel that there is a high risk of an ADR.

Besides reporting, several other factors will help to prevent ADRs. Wider use of therapeutic drug monitoring as a basis to individualise drug therapy helps to increase therapeutic efficacy and reduce ADRs. This is specially so for drugs with a small therapeutic window, and when one is treating patients at the extremes of age. Clearance, volume of distribution, and half-life can vary from patient to patient, and these variables determine the ultimate serum concentration. If the concentration reaches the toxic range ADRs are more likely to occur. Regular measurement of serum drug levels and adjusting the dose accordingly will help to minimise dose related adverse effects $(21,22,23)$.

Clinician and patient education are foremost in the prevention of ADRs. Doctors should keep abreast of recent developments in the field of therapeutics. Journals, textbooks and electronic data bases are all sources of information on ADRs (24). Drug information services, where available, play a vital role, and it should be able to pròvide the necessary information to any prescriber or patient. There is evidence that patients were responsible for $28 \%$ of the ADRs (25). This can happen from poor understanding of instructions given by the physician during consultation or by the pharmacist at time of dispensing. Patient education by physician and pharmacist will minimise such ADRs. Patients must make an effort to learn of possible ADRs of their therapy. The product information leaflet of medicines is a good source of information both to the prescriber as well as the patient.

\section{ADR reporting in Sri Lanka}

A well established ADR monitoring and reporting system is essential (26). It helps to reduce ADR-related illness in patients, and costs to the health care system. It also strengthens the arm of the national regulatory authority. Sri Lanka is now a full time member of the WHO collaboration centre for ADR Monitoring and Reporting, and the Department of Pharmacology, Faculty of Medicine, Kynsey Road, Colombo 8, is the national collaborationg centre (27). Yet the number of ADR reports received is far below what is expected. The Cosmetic Devices and Drugs Authority should initiate the statutory and logistical processes necessary to improve ADR reporting (28).

\section{References}

1. Amaiz JA, Came X, Riba N, Codina C, Ribas J, Trilla A. The use of evidence in pharmacovigilance. Case reports as the reference source for drug withdrawals. European Journal of Clinical Pharmacology 2001; 57: 89-91.

2. Edwards IR, Aronson JK. Adverse drug reactions: definitions, diagnosis, and management: Lancet 2000; 356: 1255-9.

3. deShazo RD, Kemps SF. Allergic reactions to drugs and biologic agents. Journal of the American Medical Association 1997; 278: 1895-1906.

4. Smith CC, Bennett PM, Pearce HM, Harrison PI, Reynolds DJ, et al. Adverse drug reactions in a hospital general medical unit meriting notification to the Committee on Safety of Medicines. British Joumal of Clinical Pharmacology 1996; 42: 4239.

5. Lazarou J, Pomeranz BH, Corey PN. Incidence of a adverse drug reactions in hospitalized patients: a meta-analysis of prospective studies. Journal of the American Medical Association 1998; 279: 1200-5.

6. Noah BA, Brushwood DB. Adverse drug reactions in elderly patients: alternative approaches to post-market surveillance. Joumal of Health and Law 2000; 33: 383-454.

7. Golden AG, Silverman MA, Preston RA. University of Miami division of clinical pharmacology therapeutic rounds: issues in prescribing for geriatric patients and emerging practice guidelines. American Journal of Therapeutics 1999; 6: 341-8.

8. Fattinger K, Roos M, Vergeres P, Holenstein C. Kind B, Masche $U$ et al. Epidemiology of drug exposure and adverse drug reactions in two Swiss departments of internal medicine. British Joumal of Clinical Pharmacology 2000; 49: 158-67. 
9. Ciorciaro C, Hartmann K, Kuhn M. Differences in the relative incidence of a adverse drug reactions in relation to age. An evaluation of the spontaneous reporting system of SANZ: Journal Suisse de Medicine 1998; 128: 254-8.

10. Wasserfallen JB, Francoise L, Thierry B, Laurent T, Bertrand $Y$, Jerome B. Rate, type and cost of adverse drug reactions in hospitalized patients. European Joumal of Internal Medicine 2001; 12: (in press).

11. Bordet R, Gautier S, Le Louet H, Dupuis B, Caron J. Analysis of the direct cost of adverse reactions in hospitalized patients: European Journal of Clinical Pharmacology 2001; 56: 935-41.

12. Guidelines for preparing core clinical safety information on drugs. Report of the CIOMS Working group III. Council for Intemational Organization of Medical Science, 1995.

13. Lindquist $M$, Edwards $\mathbb{R}$. The WHO programme for international drug monitoring, its database, and the technical support of the Uppsala Monitoring Centre. Joumal of Rheumatology 2001; 28: 1180-7.

14. Lindquist M, Stahl M, Bate A, Edwards IR, Meyboom RH. A retrospective evaluation of a data mining approach to aid finding new adverse drug reaction signals in the WHO international database. Drug Safery 2000; 23: 533-42.

15. Williams $D$, Feely J. Under-reporting of adverse drug reactions: attitudes of Irish doctors. Irish Journal of Medical Sciences 1999; 168: 257-61.

16. Bhattacharya SK, Singh NK, Prasad RC, Sinha PR. Some unusual and severe forms of adverse drug reactions: a call for setting adverse drug reaction monitoring centres. Journal of the Association of Physicians of India 1998; 46: 675-7.

i7. Cooper JW Jr. Consultant pharmacist drug therapy recommendations from monthly drug regimen reviews in a geriatric nursing facility: a two year study and cost analysis. Journal of Nutrition, Health and Aging 1997; 1: 181-4.

18. Moore $N$. The role of the clinical pharmacologist in the management of adverse drug reactions. Drug Safery 2001; $24: 1-7$.

19. Tully MP, Seston EM. Impact of pharmacists providing a prescription review and monitoring service in ambulatory care or community practice; Annals of Pharmacotherapy 2000; 34: 1320-31.

20. Lee A, Bateman DN, Edwards C, Smith JM, Rawlins MD. Reporting of adverse drug reactions by hospital pharmacists: pilot scheme. British Medical Joumal 1997; 315: 519.

21. Ried LD, Horn JR. Mckeena DA. Therapeutic drug monitoring reduces toxic drug reactions: a meta-analysis. Therapeutic Drug Monitor 1990; 12: 72-8.

22. Gram LF, Pedersen OL, Kristensen CB, Bjerre M, KraghSorensen P. Drug level monitoring in psychopharmacology: usefulness and clinical problems, with special reference to tricyclic antidepressants. Therapeutic Drug Monitor 1982; 4: 17-25.

23. Jelliffe RW, Schumitzky A, Van Guilder M, Liu M, Hu L, Maire $P$ et al. Individualising dng dosage regimens: roles of population pharmacokinetic and dynamic models, Bayesian fitting, and adaptive control. Therapeutic Drug Monitor 1993; 15: 380-93.

24. Morel P, Vandel B. Adverse drug reaction monitoring and the internet: evaluation of the use of the internet by French pharmacovigilance centres and a non-exhaustive survey of websites of interest for collecting information about adverse drug reactions. Therapie 1999; 54: 525-32.

25. Malhotra S, Jain S, Pandhi P. Drug-related visits to the medical department; a prospective study from India. Indian Journal of Clinical Pharmacology and Therapeutics 2001; 39: 12-8.

26. Hawton K, Townsend E, Deeks J, Appleby L, Gunnell D, Bennewitl O, Cooper, J. Effects of legislation restricting pack sizes of paracetamol and salicylate on self poisoning in the United Kingdom. British Medical Jounal 2001; 322: 1203.

27. Uppsala reports; Issue 14, April, 2001.

28. Brown SD Jr, Landry FJ. Recognizing, reporting and reducing adverse drug reactions. Southern Medical Journal 2001; 94: 370-3.

T M J Munasinghe, Pharmacologist, Department of Pharmacology, Medical Research Institute, Colombo 8. (Received 12 September 2001, accepted 10 November 2001. email: tmjanake@sltmet.lk. Telephone +941 693532) 